\title{
Experimental investigation of a novel blast wave mitigation device
}

\author{
Zhenbi Su ${ }^{\mathrm{a}}$, Wen Peng ${ }^{\mathrm{a}}$, Zhaoyan Zhang ${ }^{\mathrm{a}, *}$, George Gogos ${ }^{\mathrm{a}}$, Reed Skaggs ${ }^{\mathrm{b}}$, Bryan Cheeseman ${ }^{\mathrm{b}}$ and \\ Chian Fong Yen ${ }^{\mathrm{b}}$ \\ ${ }^{a}$ Department of Mechanical Engineering, University of Nebraska-Lincoln, Lincoln, NE 68588, USA \\ ${ }^{\mathrm{b}}$ Army Research Laboratory, Aberdeen Proving Ground, MD 21005, USA
}

Received 26 March 2007

Revised 23 July 2008

\begin{abstract}
A novel blast wave mitigation device was investigated experimentally in this paper. The device consists of a pistoncylinder assembly. A shock wave is induced within the cylinder when a blast wave impacts on the piston. The shock wave propagates inside the device and is reflected repeatedly. The shock wave propagation process inside the device lengthens the duration of the force on the base of the device to several orders of magnitude of the duration of the blast wave, while it decreases the maximum pressure over an order of magnitude. Two types of experiments were carried out to study the blast wave mitigation device. The first type of experiments was done with honeycomb structures protected by the blast wave mitigation device. Experimental results show that the device can adequately protect the honeycomb structure. A second type of experiments was done using a Hopkinson bar to measure the pressure transmitted through the blast wave mitigation device. The experimental results agree well with results from a theoretical model.
\end{abstract}

Keywords: Shock wave, mitigation device, Hopkinson bar

\section{Nomenclature}

$$
\begin{aligned}
& A=\text { cross-sectional area of piston } \\
& D=\text { diameter of piston } \\
& e=\text { coefficient of restitution } \\
& I=\text { impulse per unit area } \\
& L=\text { length of cylinder } \\
& m=\text { mass } \\
& t=\text { time } \\
& u=\text { incident velocity of pendulum ball } \\
& x=\text { Cartesian coordinate } \\
& \delta=\text { thickness of piston } \\
& \text { Subscript } \\
& b=\text { pendulum ball } \\
& p=\text { piston }
\end{aligned}
$$

${ }^{*}$ Corresponding author. Tel.: +1 402472 4993; Fax: +1 402472 1465; E-mail: zzhang5@unl.edu. 


\section{Introduction}

A blast wave produced by an explosive can be very damaging to military personnel and structures. Extensive research has been conducted to reduce the damage potential of blast waves. The damage potential of an explosive blast depends on three main factors, the force exerted on the target, the duration of the applied force and the ability of the target to withstand the effects of the blast wave [7]. There are two main approaches, blast absorbing materials and heterogeneous systems, to mitigate the damage potential of the blast wave.

Water has been used to reduce the damage caused by blast waves. Gelfand and Silnikov [5] proposed an attenuation method which uses a liquid layer confined within an elastic envelop to mitigate the blast wave. Their results showed that the blast wave pressure attenuation coefficient depends on the distance from the blast epicenter to the point of measurement as well as the thickness of the water layer. Shin et al. [14] numerically simulated the mitigation effects of watershield on air blast wave. The magnitude of peak pressure and the shock wave arrival time were analyzed. The results showed that water medium delays the shock front and reduces the magnitude of initial peak shock pressure by $40 \%$. Chong et al. [2] numerically simulated the blast mitigation process by water using a three-dimensional model. The peak pressures on the target with and without water have been obtained and analyzed. Results from their numerical simulations showed good agreement with experimental data for cases with and without water. The peak pressure can be reduced by more than $50 \%$ with water in comparison with the case without water.

Foams including aqueous foams and metal foams were used to mitigate the blast wave. Panczak et al. [11] studied the blast wave mitigation of aqueous foams. They concluded that vaporization of the liquid component was detrimental to blast wave mitigation. It was noted that many reflections off the foam/air interface produce a complicated waveform in the aqueous foam. The impedance mismatch between the air and foam is an important factor for small foam depth, while the impedance mismatch has little influence for large foam depth. Li and Meng [8] investigated the blast mitigation behavior of cellular materials by using a one-dimensional mass-spring model. It was indicated that the transmitted pressure can be attenuated by the foam layer if the input blast load is below a critical value. This material can be used only for the lower pressure blast wave. For the high blast wave pressure, the cellular material will be destroyed and the pressure on the target will increase badly. Full-scale field tests have been carried out by Hanssen et al. [6] to investigate the behavior of aluminum foam panels subjected to blast loading. Foam panels were attached to the bob of a ballistic pendulum. The energy and impulse transfer from the blast loading can be calculated through the maximum swing of the pendulum. An analytical solution was proposed to analyze the experiments and results showed that surface effects can affect the energy and impulse transfer.

The list of blast absorbing materials typically includes granular, particulate matter, porous material, foam and water. Granular, particulate matter, porous material and foam were studied by Nesterenko [9]. Different materials used to mitigate blast waves were analyzed. The momentum and energy of a blast wave can be absorbed by these "soft" condensed matters. The density, porosity and relative geometrical size of the so-called "soft" condensed matter are the main parameters determining the effectiveness of blast wave mitigation. Pfannes et al. [10] investigated a tapered chain of elastic beads under impulse loading. The elastic beads act as an absorber of kinetic energy and can reduce it by about $30 \%$. Results show that the energy absorption is affected by the restitution coefficient, the size of the particles and the tapering ratio. Edwards and Palmer [4] carried out experiments to determine the blast mitigation characteristics of particulate materials. They employed particulate materials with different densities, sizes and distributions as a mitigation layer. Results showed that the mitigation of an explosion is enabled largely by the consolidation of low density particulate matter into compacts of greater density. Mitigation effects decrease with average particulate size for particulate material with low areal densities.

Heterogeneous systems have also been designed to attenuate blast waves. Chapman et al. [3] examined the effect of geometrical parameters of a blast wall to protect a target structure. The authors used one-tenth scale to assess the sensitivity of blast parameters and measured the overpressure of the target behind the blast wall. The results showed the relationship between blast mitigation and geometrical configuration of the wall. This relationship can be used to optimize the parameters of the blast wall. Rose et al. [12,13] measured the overpressure behind a one-tenth scale vertical blast wall. The height and distance of the wall affect the mitigation effectiveness. Results showed that the overpressures behind the wall are $30 \%$ to $60 \%$ of those without a wall. Su et al. [15] developed a numerical model of a novel blast wave mitigation device which consists of a piston-cylinder assembly. Through the shock wave propagation and reflection inside the blast wave mitigation device, the blast wave can be mitigated. The peak 
B last W ave

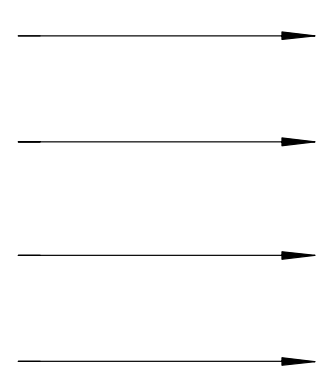

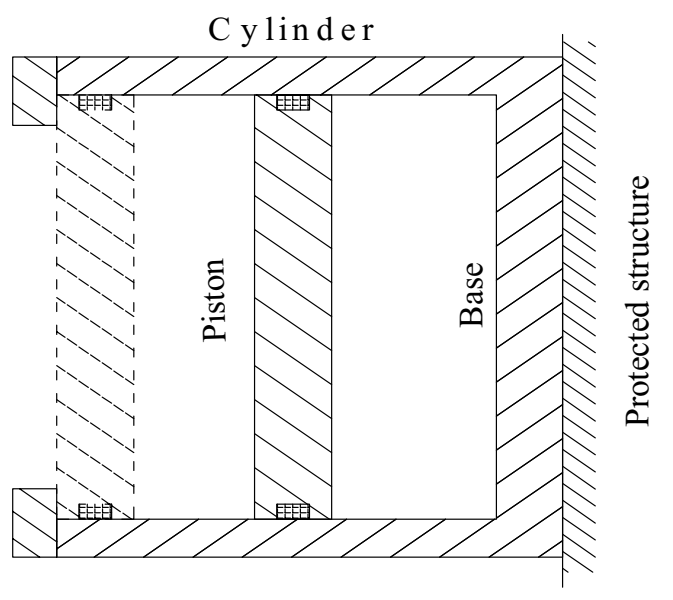

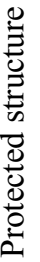

Fig. 1. Schematic of the blast wave mitigation device.
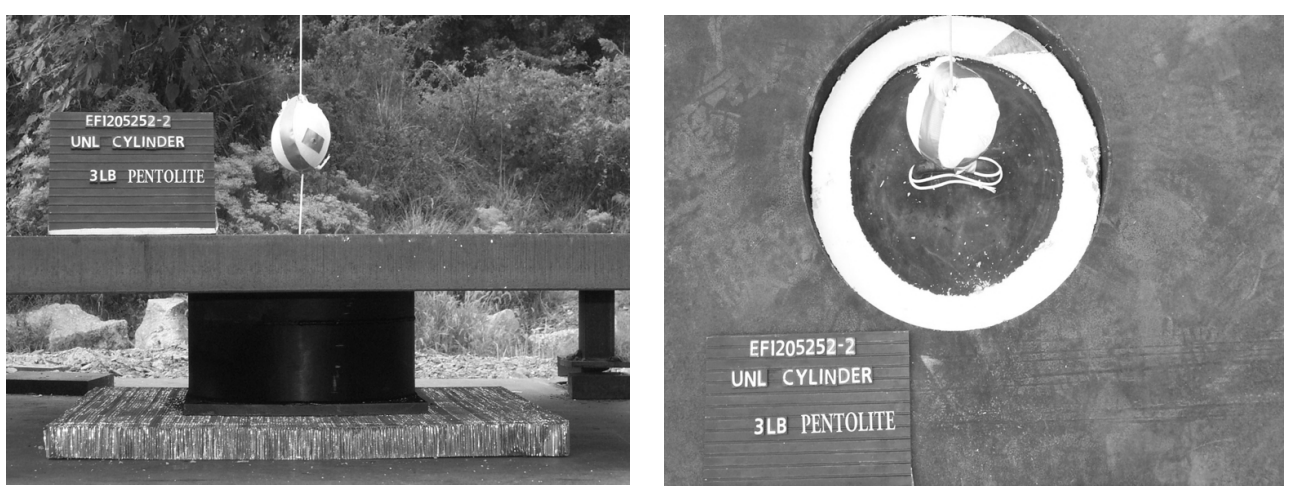

Fig. 2. The first experimental setup for the explosive charge test.

pressure of the blast wave can be reduced by $95 \%$. This blast wave mitigation device is effective for the mitigation of blast waves generated by high energy explosion.

In this paper, the blast wave mitigation device proposed in [15] is investigated experimentally for the first time. The device consists of a piston-cylinder assembly, which is shown in Fig. 1. The piston is sealed by an o-ring. In this study, air is being compressed within the device. A shock wave is induced inside the device when it is subject to a blast wave. The shock wave propagates inside the device and is reflected repeatedly. The shock wave propagation process inside the device lengthens the duration of the force on the base of the device to several orders of magnitude of the duration of the blast wave, while it decreases the maximum pressure over an order of magnitude, depending on the design parameters chosen. It should be noted that the piston-cylinder assembly has been used in dampers in many engineering systems. However, there are significant differences between physical mechanisms involved in the piston damper and the blast wave mitigation device. The piston dampers are used to diminish mechanical vibration. There is a blow-off valve to modulate the pressure inside the damper. The physical process involved is an equilibrium process over a long duration. Viscosity of the fluid inside the piston damper plays a significant role. In the blast wave mitigation device, because there is no blow-off valve, the viscosity of the fluid is not important for the blast wave mitigation device. The pressure on the base of the blast wave mitigation device is the key parameter determining the effectiveness of the device. The lower pressure implies better effectiveness of the device. A continuous grid of the proposed device can be used to cover the surface of military structures, to significantly reduce personnel casualties and equipment damage. In this paper, experiments were carried out to study the blast wave mitigation device. 


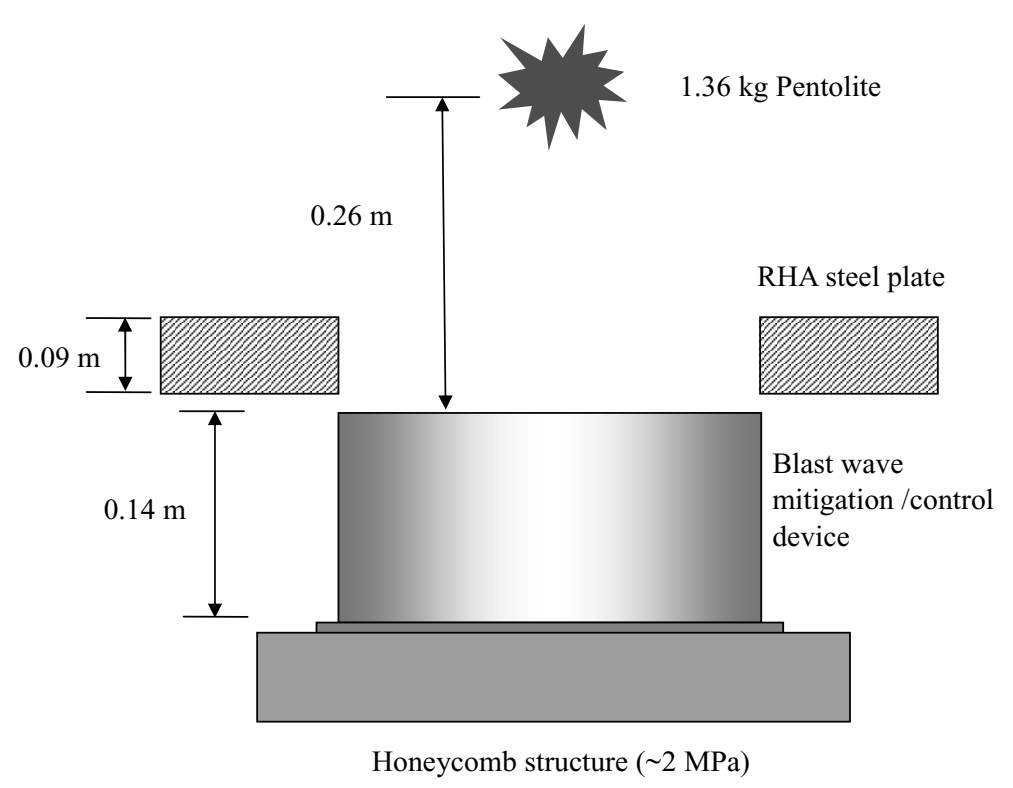

Fig. 3. Schematic of the first experimental setup for the explosive charge test.
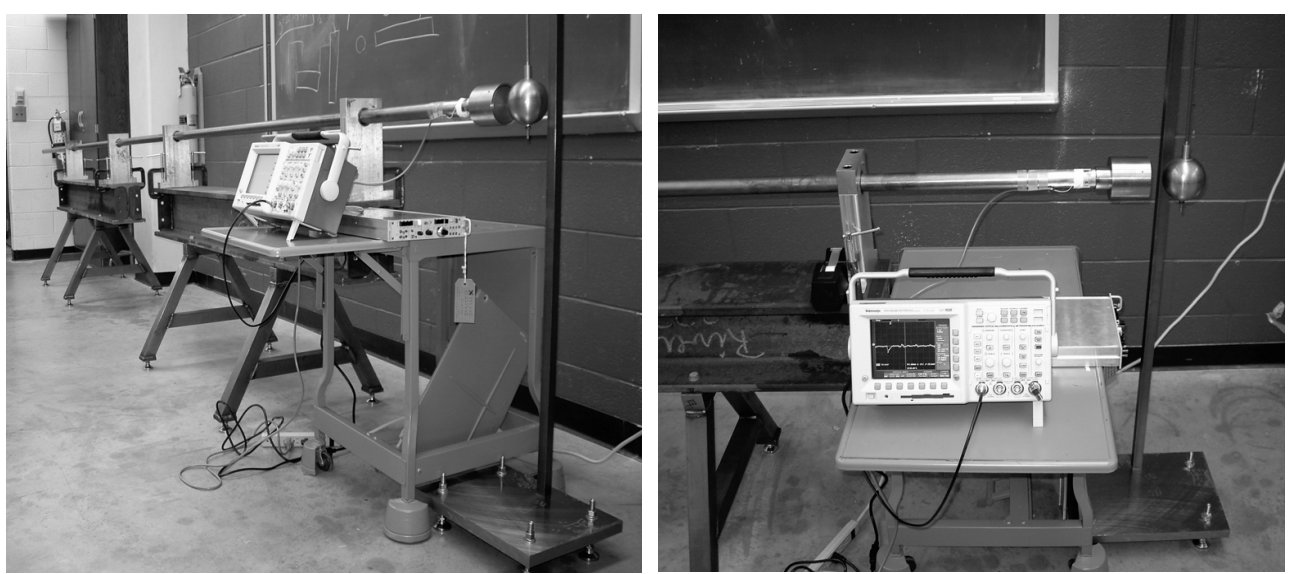

Fig. 4. The second experimental setup consisting of a Hopkinson bar and a ball pendulum.

\section{Experimental approach}

Two sets of experiments were carried out to study the effectiveness of the blast wave mitigation device. The first set of experiments was set up at the Army Research Laboratory (ARL), as shown in Fig. 2. The schematic of the first experimental setup is shown in Fig. 3. A honeycomb structure, which can withstand a peak pressure of $2 \mathrm{MPa}$ (with a safety factor of about 2), is placed on a steel test platform (not shown). A blast wave mitigation device or a control device is placed on top of the honeycomb structure. The control device has the same dimensions and weight as the blast wave mitigation device. However, the piston and the cylinder of the control device are welded together. The control device has no moving part. Therefore, there is no induced shock wave within the control device. As a result, the blast wave can be transmitted through the control device with negligible attenuation. A Rolled Homogeneous Armor (RHA) steel plate is used to protect the honeycomb structure that is not covered by the blast wave mitigation/control device. A hole was cut at the center of the RHA steel plate to expose the blast wave mitigation/control device. The diameter of the hole is the same as that of the blast wave mitigation/control device. 


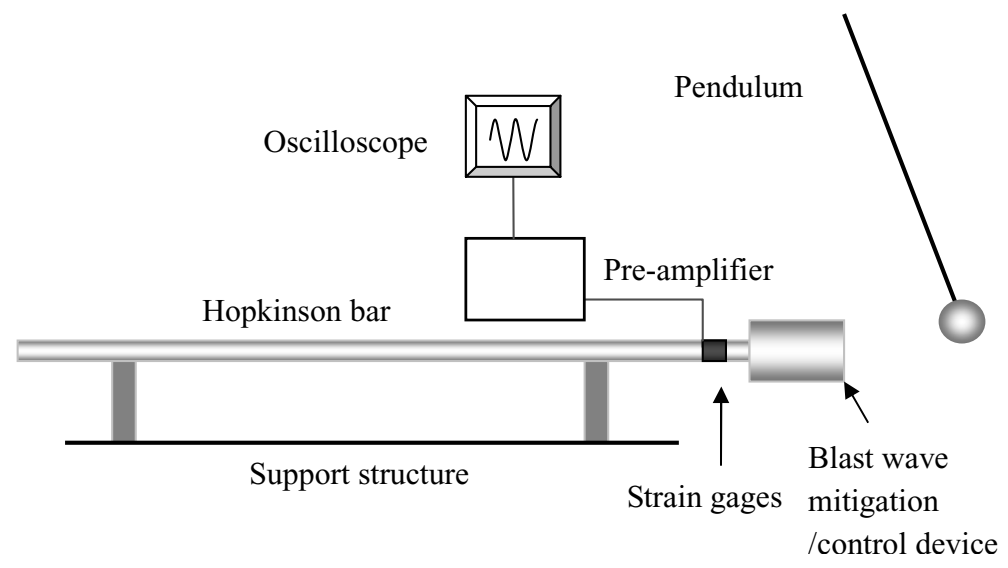

Fig. 5. Schematic of the second experimental setup consisting of a Hopkinson bar and a ball pendulum.

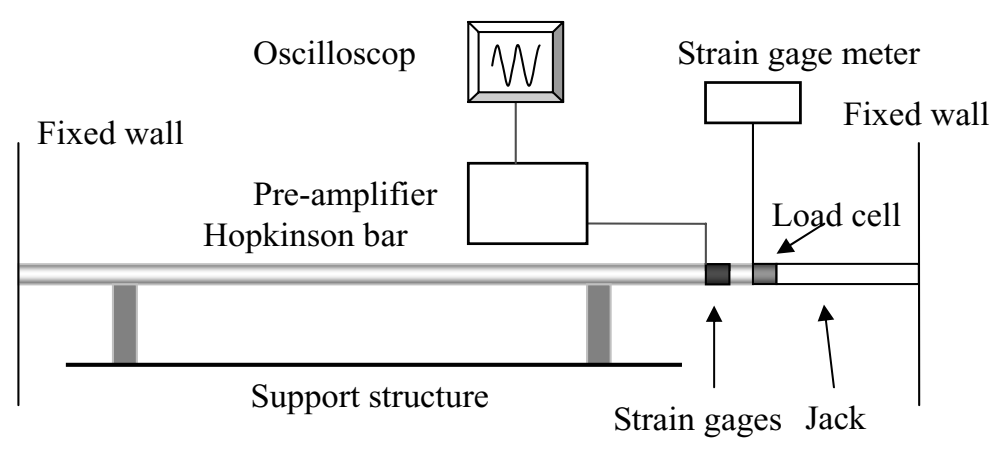

Fig. 6. Schematic for the Hopkinson bar calibration.

The RHA steel plate has a thickness of $9 \mathrm{~cm}$. The blast wave is generated by detonating $1.36 \mathrm{~kg}$ of Pentolite at a distance of $0.26 \mathrm{~m}$.

The second set of experiments was set up at the University of Nebraska-Lincoln, as shown in Fig. 4. The schematic of the second experimental setup is shown in Fig. 5. The setup mainly consists of a steel Hopkinson bar and a ball pendulum. The Hopkinson bar mainly includes a steel bar $6.15 \mathrm{~m}$ in length and $0.038 \mathrm{~m}$ in diameter. The distance of the strain gages from the far end of the Hopkinson bar is roughly $6 \mathrm{~m}$. When a pendulum ball strikes the right end of the steel bar, a compression wave propagates along the bar. It is reflected at the left end. A smaller set of blast wave mitigation/ control device, which is specifically designed for the Hopkinson bar experiments, is attached on the right end of the Hopkinson bar. The interface of the Hopkinson bar and the blast wave mitigation/control device has been grinded. In addition, lubricant is used at the interface to ensure good contact. A full Wheatstone bridge with four strain gages (OMEGA SG-6/12-LY11) is applied to the right end of the Hopkinson bar to measure the strain. A pre-amplifier (VISHAY 2310B) is used to excite the Wheatstone bridge and amplify the electrical signal from the Wheatstone bridge. The amplified electrical signal is displayed and stored on an oscilloscope (Tektronix TDS3054B). A ball pendulum is used to generate an impact that simulates the impact due to a blast wave. The mass of the pendulum ball is $1.68 \mathrm{~kg}$ and it falls freely from a height of $1.62 \mathrm{~m}$. The ball reaches an incident velocity of $u=5.6 \mathrm{~m} / \mathrm{s}$ upon impact.

In order to translate the electrical signal from the Wheatstone bridge to pressure, the Hopkinson bar has been calibrated. Figure 6 shows the schematic of the calibration. The Hopkinson bar is clamped by two fixed walls. A constant force is generated by a hydraulic jack. A calibrated load cell (OMEGA LC401-10K), together with a strain gage meter (OMEGA DP41-S), is used to measure the force loaded on the Hopkinson bar, while the electrical signal from the Wheatstone bridge is recorded. The calibration is conducted at several force levels. A maximum force of 13,500 $\mathrm{N}$ was used. The Hopkinson bar becomes unstable beyond this value. The experimental data are shown 
Table 1

Parameters of the blast wave mitigation device used with the ARL experiment

\begin{tabular}{ccc}
\hline \multicolumn{2}{c}{ Piston(steel) } & $\begin{array}{c}\text { Cylinder(steel) } \\
L(\mathrm{~mm})\end{array}$ \\
\cline { 1 - 2 }$\delta(\mathrm{mm})$ & $D(\mathrm{~mm})$ & 140 \\
\hline 50 & 420 & \\
\hline
\end{tabular}

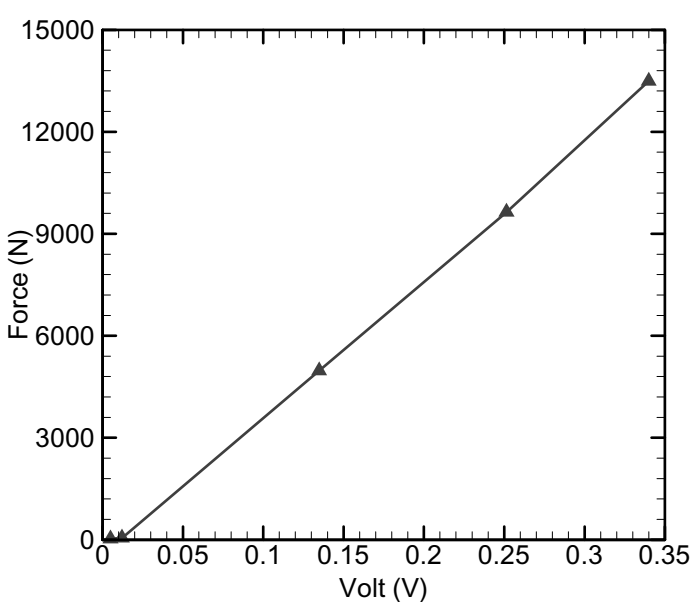

Fig. 7. Hopkinson bar force versus electrical signal.

in Fig. 7. The curve is almost a straight line. Using the least mean square fitting, it is determined that the ratio of the force to the electrical signal is $40.8 \mathrm{kN} / \mathrm{V}$. Since the Hopkinson bar is capable of measuring the blast wave transmitted through the blast wave mitigation device as a function of time, it can be used to test the effectiveness of the blast wave mitigation device quantitatively.

\section{Results and discussion}

The first set of experiments was conducted at ARL using the honeycomb structure. The design parameters of the blast wave mitigation device used with the honeycomb structure are listed in Table 1. The parameters that significantly affect the effectiveness of the blast wave mitigation device are the thickness of the piston $\delta$ and the length of the cylinder $L . D$ is the diameter of the piston. The control device has the same dimensions and weight as the blast wave mitigation device. The blast wave mitigation/control device has a square base of $460 \times 460 \times 12.5$ $\mathrm{mm}$. One experiment was conducted with the control device and one with the blast wave mitigation device. The blast wave corresponds to an explosion generated by $1.36 \mathrm{~kg}$ Pentolite, which is $0.26 \mathrm{~m}$ away from the end front of the blast wave mitigation/control device. As the blast wave impacts the blast wave mitigation/control device, the blast wave is reflected. Using the CONWEP program, which is an analytical curve-fit based on experimental results, the peak reflected pressure exerted on the piston of the blast wave mitigation/control device is roughly $140 \mathrm{MPa}$. The duration of the reflected pressure is roughly $0.2 \mathrm{~ms}$. The reflected pressure obtained from the CONWEP program as a function of time is shown in Fig. 8. When the Pentolite is detonated, the blast wave can be transmitted through the control device with negligible attenuation. Since the blast wave pressure significantly exceeds the pressure rating of the honeycomb structure, not surprisingly, the impact of the blast wave leaves an indentation of roughly $13 \mathrm{~mm}$ in the honeycomb structure, when the control device is used (see Fig. 9).

When the blast wave mitigation device is used, the impact of the blast wave causes the piston to recede. The piston movement induces a weak shock wave inside the blast wave mitigation device. The shock wave propagates inside the blast wave mitigation device and is reflected repeatedly. Each time, the shock wave is reflected, the pressure, temperature and density increase. The increased pressure slows down the piston, which eventually comes 


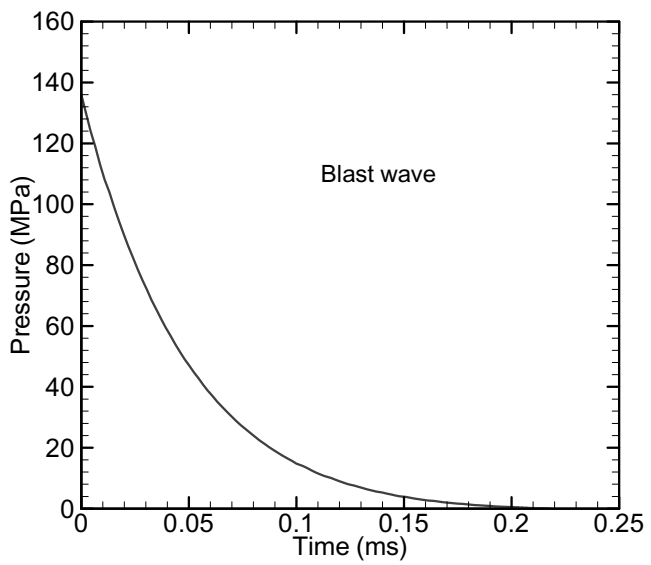

Fig. 8. Reflected pressure exerted on the blast wave mitigation/control device.

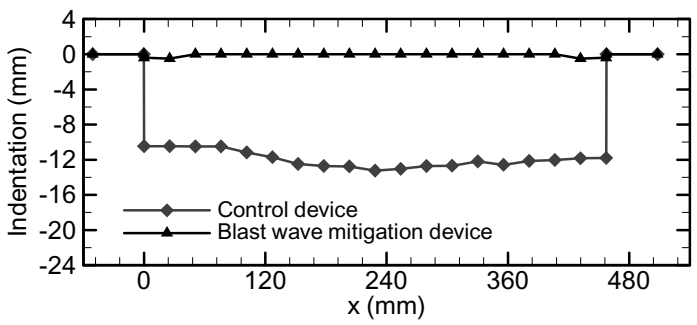

Fig. 9. Honeycomb indentations with the control device and the blast wave mitigation device.

to a complete stop. At this moment, the pressure on the base of the device reaches its maximum. The shock wave propagation process inside the device lengthens the duration of the force on the base of the device to several orders of magnitude of the duration of the blast wave. While the impulse of the blast wave is almost conserved, this results in decrease of the maximum pressure over an order of magnitude. A numerical model was developed [15] that can predict the pressure of the blast wave transmitted through the device. The Van Leer flux vector splitting method coupled with the monotone upstream-centered scheme for conservation laws (MUSCL) and Runge-Kutta scheme was applied to solve the Euler equations governing the shock wave propagation process within the blast wave mitigation device. Because the shock wave in the flow fields introduces sharp gradients, such a discontinuous change always has the potential to cause oscillations in the numerical solutions. A standard upwind scheme can provide sufficient numerical dissipation for solving the present problem. However, the directions of characteristic velocity should be established first, before the upwind scheme can be implemented. The Van Leer flux vector splitting scheme was applied to split the flux vector into forward and backward components. The forward and backward flux components have characteristic velocities going forward and backward, respectively. The spatial derivatives of the flux vectors were approximated with backward and forward differencing using MUSCL. MUSCL is a first order upwind scheme in the vicinity of the shock wave and second order upwind scheme elsewhere in the flow field. It provides good stability as well as accuracy. A two-step Runge-Kutta explicit scheme was used to solve the Euler equations. The detailed numerical scheme and solution algorithm have been described in [15]. Using the numerical model, the effects of some important design parameters, including the mass of the piston and the cylinder length, on the blast wave mitigation were studied. As the mass of the piston increases or as the cylinder length increases, the pressure on the base of the device is reduced by a larger percentage The essence of the mitigation device is to reduce a high-pressure short-duration impact to a low-pressure long-duration impact. The reflected pressure profile, as shown in Fig. 8, is used as input data to the numerical model, which is developed in [15]. The peak pressure on the base of the blast wave mitigation device, predicted by the numerical simulation, is about $3 \mathrm{MPa}$, as shown in Fig. 10. The reduction of the peak blast wave pressure is predicted to be over 97\%. As a result, the honeycomb structure should be adequately protected by the blast wave mitigation device. This was confirmed experimentally; 
Table 2

Parameters of the blast wave mitigation devices used with the Hopkinson bar experiments

\begin{tabular}{cccc}
\hline & \multicolumn{2}{c}{ Piston(steel) } & Cylinder(steel) \\
\cline { 2 - 3 } & $\delta(\mathrm{mm})$ & $D(\mathrm{~mm})$ & $L(\mathrm{~mm})$ \\
\hline $\mathrm{A}$ & 12.5 & 80 & 37 \\
$\mathrm{~B}$ & 14 & 80 & 50 \\
\hline
\end{tabular}

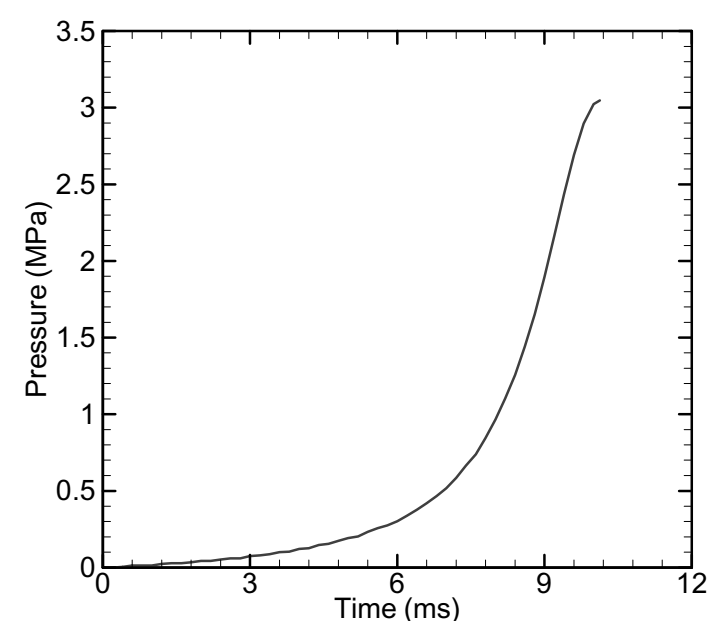

Fig. 10. Predicted pressure on the base of the blast wave mitigation device.

the blast wave impact only leaves very shallow indentation along the edge of the device, which is where the stress concentration occurs. The indentations on the honeycomb structures with the blast wave mitigation device and the control device under the impact of the blast wave are shown in Fig. 9. The experimental results prove qualitatively that the blast wave mitigation device is effective in mitigating the blast wave impact.

The second set of experiments was conducted at the University of Nebraska-Lincoln (UNL) using the Hopkinson bar experimental setup described above. Two new blast wave mitigation devices were designed and built to fit the Hopkinson bar experiment. A pendulum ball is used to generate an impact to simulate the impact of a blast wave. Pressures on the bases of the blast wave mitigation devices as a function of time are measured and recorded. The parameters of the two different blast wave mitigation devices, A and B, are listed in Table 2. The thickness of the piston and the length of the cylinder are key parameters of a blast wave mitigation device. Thicker piston results in smaller shock wave strength. Longer cylinder leads to longer duration for shock wave propagation. The pressure at the base of the cylinder increases at a slower rate when the piston is thicker and the cylinder is longer. Therefore, the blast wave mitigation device $\mathrm{B}$ is expected to be more effective than the blast wave mitigation device A.

In addition, control devices with the same dimensions and masses as the blast wave mitigation devices have been designed and built. Again, the piston of the control device is welded to the cylinder. There is no induced shock wave within the control device. Hence, there is no blast wave mitigation mechanism. As a result, the blast wave can be transmitted through the control device with negligible attenuation. The experiment results using the control device are measured and compared with that using the corresponding blast wave mitigation device. The control devices can also be used to further check the calibration of the Hopkinson bar using the same pendulum set. Theoretically, the impact of the pendulum ball on the control device generates an impulse of $1887 \mathrm{~Pa} \cdot \mathrm{s}$ on the control device. The impact of the pendulum ball is measured by the Hopkinson bar and integrated. The process yields the impulse on the control device, which averages $1773 \mathrm{~Pa} \cdot \mathrm{s}$. The difference between the theoretical and experimental results is roughly $6 \%$, which indicates that the Hopkinson bar is properly calibrated.

Experiments using the blast wave mitigation device were carried out using the Hopkinson bar. A pendulum ball strike is used to simulate the impact of the blast wave. The interaction between the blast wave mitigation device and the pendulum ball is actually more complex than that between the device and the blast wave. In order to compare the 


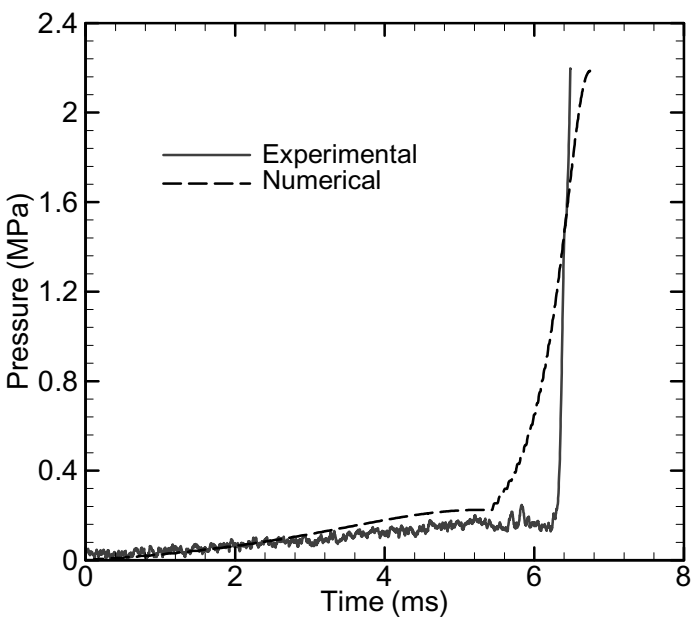

Fig. 11. Comparison of measured pressure on the base of device A (obtained from the Hopkinson bar test) with numerical prediction.

experimental results with a numerical model, a double-collisions model is used to calculate the impulse delivered to the blast wave mitigation device from the pendulum ball. The first collision between the pendulum ball and the device is partially inelastic. The velocities of the piston and the pendulum ball after the collision are [1]

$$
\begin{aligned}
& u_{p}=\frac{(1+e) m_{b}}{m_{b}+m_{p}} u \\
& u_{b}=\frac{m_{b}-e m_{p}}{m_{b}+m_{p}} u
\end{aligned}
$$

where $u_{p}$ and $u_{b}$ are the velocities of the piston and the pendulum ball, $m_{p}$ and $m_{b}$ are the mass of the piston and the pendulum ball and $e$ is the coefficient of restitution between the piston and the pendulum ball. The impulse per unit area delivered to the piston is

$$
I_{p}=m_{p} u_{p} / A
$$

where $A$ is the cross-sectional area of the piston. After the first collision, the piston and the pendulum ball move with different velocities toward the base of the blast wave mitigation device. The shock wave generated inside the blast wave mitigation device increases the pressure inside the device. The increasing pressure reduces the velocity of the piston. The motion of the piston and the shock propagation process has been simulated by a numerical model [15]. The pendulum ball, however, moves with a constant velocity of $u_{b}$. After a short period of time, the pendulum ball catches up with the piston. A second collision occurs. The position and time of the second collision can be calculated using the numerical model. The process repeats until the piston comes to a complete stop. To simplify the collision process, it is assumed that there are only two collisions between the pendulum ball and the device. The second collision is considered to be fully inelastic collision. As a result, the piston and the pendulum ball move with the same velocity. The impulse that the piston obtains through the second collision is

$$
I_{p}=m_{b} u_{b} / A
$$

Figure 11 shows the comparison of the numerical and the experimental pressure on the base of the blast wave mitigation device A. In the numerical model, a coefficient of the restitution $e$ between the pendulum ball and the piston is assumed to be 0.3 . After the first collision, the piston has a velocity of $5.67 \mathrm{~m} / \mathrm{s}$ while the pendulum ball has a velocity of $3.98 \mathrm{~m} / \mathrm{s}$. The impulse delivered to the piston is $557.2 \mathrm{~Pa} \cdot \mathrm{s}$. The impact causes the pressure inside the blast wave mitigation device to increase gradually. The pressure inside the blast wave mitigation device reduces the velocity of the piston. At approximately $5.2 \mathrm{~ms}$, the pendulum ball catches up with the piston and the second collision occurs. The second collision delivers to the piston an impulse of $1329.8 \mathrm{~Pa} \cdot \mathrm{s}$, which causes the piston to move with a higher velocity. High velocity induces a shock wave with strong shock wave strength. Therefore, the 


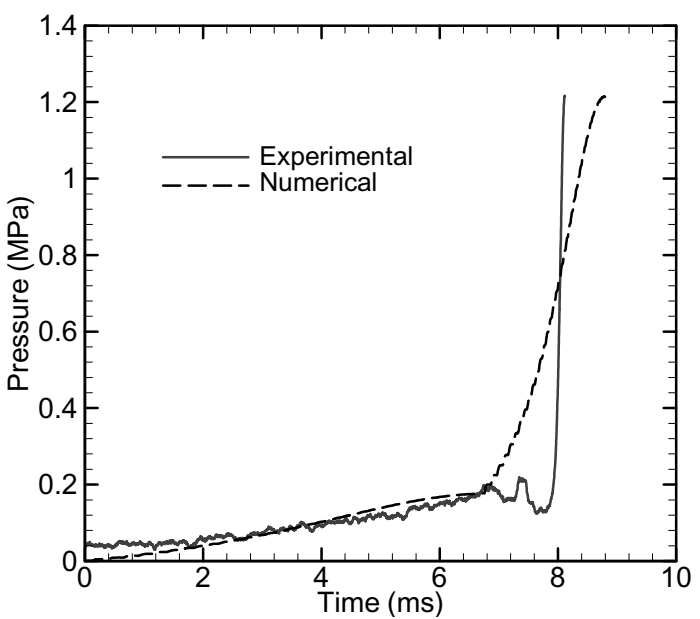

Fig. 12. Comparison of measured pressure on the base of device B (obtained from the Hopkinson bar test) with numerical prediction.

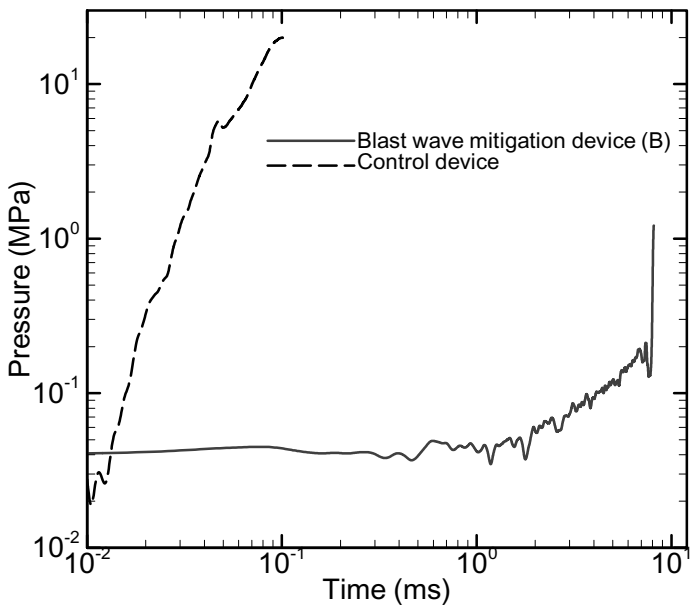

Fig. 13. Pressure obtained from the Hopkinson bar test on the base of the blast wave mitigation device (B) and of the corresponding control device.

pressure inside the blast wave mitigation device increases at a faster rate. At about $6.8 \mathrm{~ms}$, the piston comes to a complete stop. The pressure on the base of the blast wave mitigation device reaches its maximum value of 2.185 $\mathrm{MPa}$, which compares very favorably to the measured peak pressure of $2.19 \mathrm{MPa}$. The experimental results agree well with the numerical results during the first $5 \mathrm{~ms}$. The pressure increases very rapidly toward the end of the piston motion. The experimental rate of increase is significantly higher than that predicted by the numerical model. The discrepancy is likely due to the fact that the numerical model uses a double collisions model, while the actual interaction between the pendulum ball and the piston involves a series of collisions. Another fact that may contribute to the discrepancy is that experiments last 6 to $8 \mathrm{~ms}$, while the Hopkinson bar is capable of recording clean signal of $2.4 \mathrm{~ms}$, which can be measured by the oscilloscope. During the later stage of the experiment, some small reflected stress wave may distort the stress curve.

Figure 12 shows the comparison of the numerical and the experimental pressure on the base of blast wave mitigation device B. After the first collision, the piston has a velocity of $5.52 \mathrm{~m} / \mathrm{s}$ while the pendulum ball has a velocity of $3.83 \mathrm{~m} / \mathrm{s}$. The impulse delivered to the piston is $607.5 \mathrm{~Pa} \cdot \mathrm{s}$. The pressure inside the blast wave mitigation device increases gradually due to the impact. The pendulum ball catches up with the piston and the second collision occurs at approximately $7.2 \mathrm{~ms}$. The piston obtains an impulse of $1279.5 \mathrm{~Pa} \cdot \mathrm{s}$ through the second collision. At about $8.6 \mathrm{~ms}$, the piston comes to a complete stop and the peak pressure on the base of the blast wave mitigation device 
reaches 1.214 $\mathrm{MPa}$, which agrees well with the experimental peak pressure of 1.22 $\mathrm{MPa}$. Similarly to the results for device A in Fig. 11, the numerical results closely follow the experimental results up to roughly 7 ms. The numerical model underpredicts the rate of increase towards the end of the process. The pressure on the base of the cylinder both for the control device and for the blast wave mitigation device B is shown in Fig. 13. It can be seen clearly that the peak pressure experienced at the base of the blast wave mitigation device can be smaller than that of the control device by over an order of magnitude, while the duration of the former is increased by orders of magnitude compared to the duration of the latter.

\section{Conclusion}

In this work, the effectiveness of a proposed blast wave mitigation device has been investigated through two types of experiments. The first set of experiments uses a honeycomb structure to test the effectiveness of the blast wave mitigation device qualitatively. Experimental results show that the blast wave pressure can be reduced by the blast wave mitigation device significantly. The second set of experiments uses the Hopkinson bar to measure the pressure on the base of the blast wave mitigation device as a function of time. A pendulum ball strike is used to simulate the blast wave impact. This setup is capable of testing the effectiveness of the blast wave mitigation device quantitatively. Experimental results show that the peak pressure of the blast wave can be reduced by more than $90 \%$ by the proposed blast wave mitigation device. Numerical results are compared with the experimental results. They agree reasonably well. The numerical model has been validated and can be used to optimize the design of the blast wave mitigation device in the future.

\section{Acknowledgments}

Financial support for this study by Army Research Office Contract W911NF-04-2-0011 is gratefully acknowledged.

\section{References}

[1] T. Baumeister, E.A. Avallone and T. Baumeister, III., Standard Handbook for Mechanical Engineers, (8th ed.), McGraw-Hill, 1978.

[2] W.K. Chong, K.Y. Lam, K.S. Yeo, G.R. Liu and O.Y. Chong, Comparison of Simulation's Results with Experiment on Water Mitigation of an Explosion, Shock and Vibration 6(2), 1999, 73-80.

[3] T.C. Chapman, T.A. Rose and P.D. Smith, Reflected Blast Wave Resultants behind Cantilever Walls: A New Prediction Technique, International Journal of Impact Engineering 16(3) (Jun. 1995), 397-403.

[4] M.R. Edwards and M.E. Palmer, Mitigation of Comminution Effects of Explosives by Particulate Materials, Journal of Applied Physics 93(5) (Mar. 2003), 2540-2543.

[5] B.E. Gelfand and M.V. Silnikov, The Selection of the Effective Blast Reduction Method when Detonating Explosives, Journal de Physique IV: JP 12(7) (Aug. 2002), Pr7/371-Pr7/374.

[6] A.G. Hanssen, L. Enstock and M. Langseth, Close-range Blast Loading of Aluminium Foam Panels, International Journal of Impact Engineering 27(6) (Jul. 2002), 593-618.

[7] G.F. Kinney and K.J. Graham, Explosive Shocks in Air, (2nd ed.), Springer-Verlag New York, 1985.

[8] Q.M. Li and H. Meng, Attenuation or Enhancement -A One-dimensional Analysis on Shock Transmission in the Solid Phase of a Cellular Material, International Journal of Impact Engineering 27(10) (Nov. 2002), 1049-1065.

[9] V.F. Nesterenko, Shock Mitigation by 'Soft' Condensed Matter, Materials Research Society Symposium-Proceedings 759, $2003,135-146$.

[10] J. Sen Pfannes, S. Chakravarti and F.I. Surve, Energy Absorption and Recovery in Tapered Granular Chains: Small Chains and Low Tapering, Materials Research Society Symposium-Proceedings 759, 2003, 147-154.

[11] T.D. Panczak, H. Krier and P.B. Butler, Shock Propagation and Blast Attenuation through Aqueous Foams, Journal of Hazardous Material 14(3) (Mar. 1987), 321-336.

[12] T.A. Rose, P.D. Smith and G.C. Mays, The Effectiveness of Walls Designed for the Protection of Structures against Airblast from High Explosives, Proceedings of the Institution of Civil Engineers, Structures and Buildings 110(1) (Feb. 1995), 78-85.

[13] T.A. Rose, P.D. Smith and G.C. Mays, Protection of Structures against Airblast using Barriers of Limited Robustness, Proceedings of the Institution of Civil Engineers, Structures and Buildings 128(2) (May 1998), 167-176.

[14] Y.S. Shin, M. Lee, K.Y. Lam and K.S. Yeo, Modeling Mitigation Effects of Watershield on Shock Waves, Shock and Vibration 5(4) (1998), 225-234.

[15] Z. Su, W. Peng, Z. Zhang, G. Gogos, R. Skaggs and B. Cheeseman, Numerical Simulation of a Novel Blast Wave Mitigation Device, International Journal of Impact Engineering 35(5) (Feb. 2008), 336-346. 

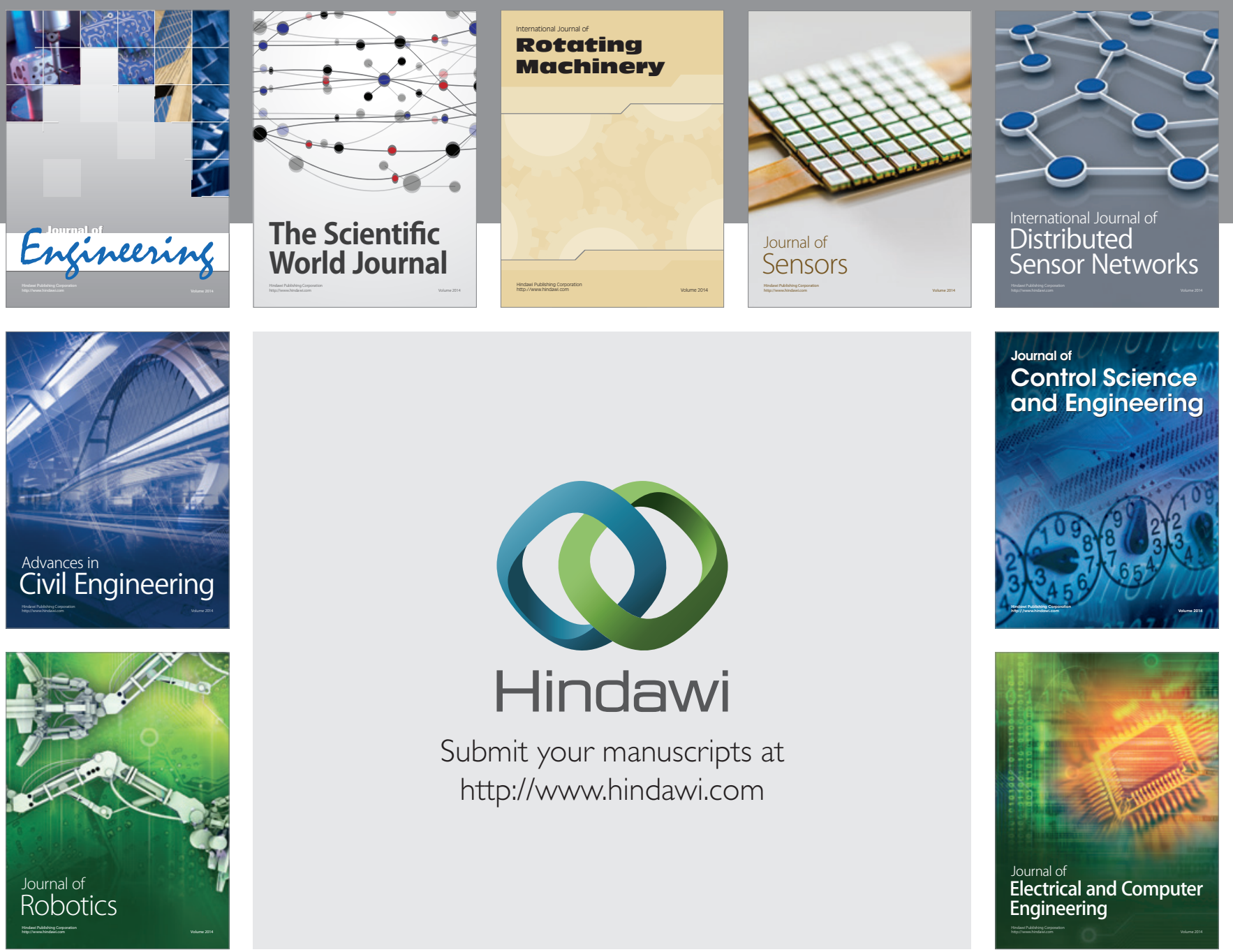

Submit your manuscripts at

http://www.hindawi.com
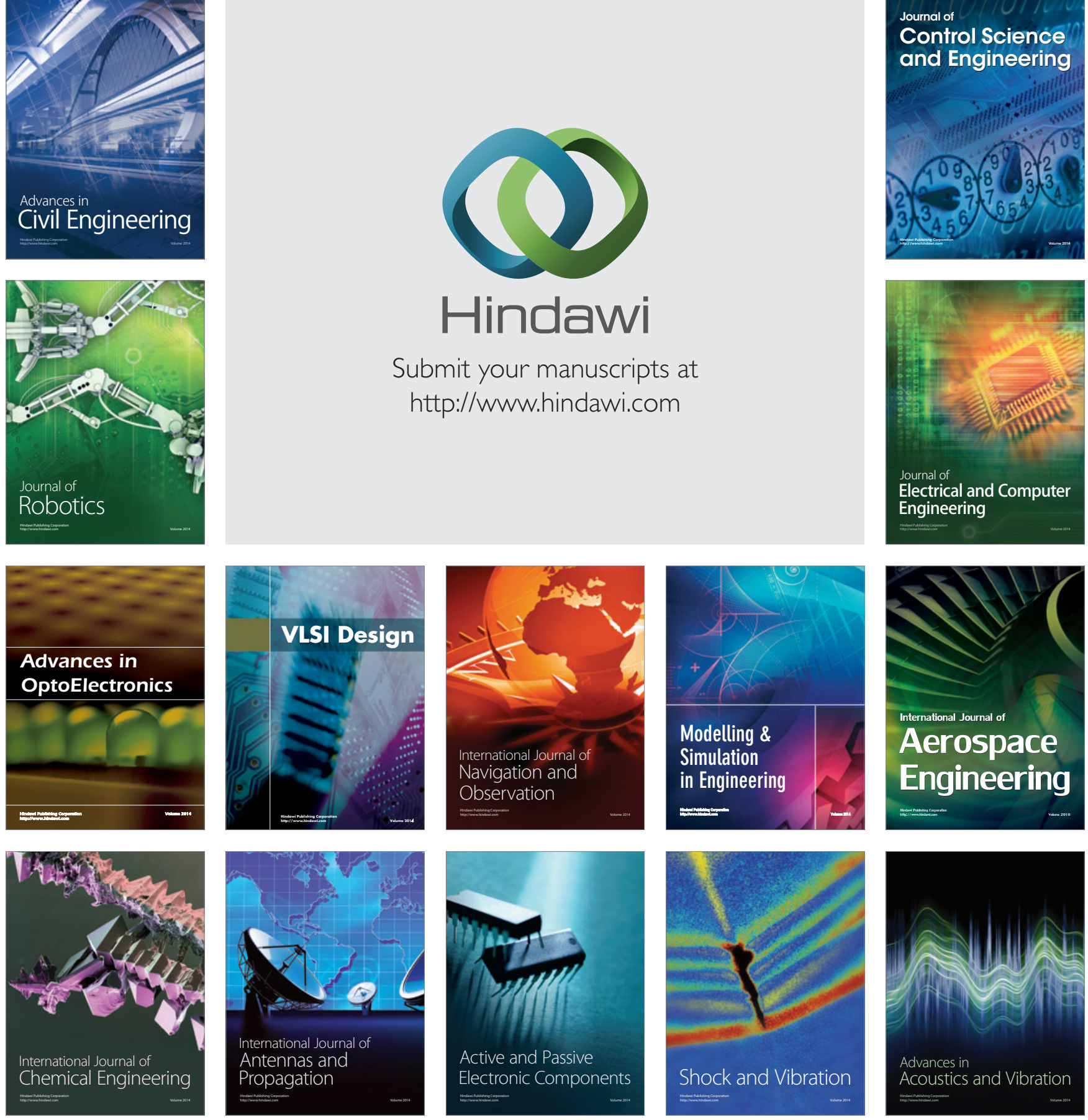